ORIGINAL ARTICLE

\title{
Fitness levels of middle aged martial art practitioners
}

\author{
P Douris, A Chinan, M Gomez, A Aw, D Steffens, S Weiss
}

Br J Sports Med 2004;38:143-147. doi: 10.1136/bjsm.2002.001768

See end of article for authors' affiliations .....................

Correspondence to: Dr Douris, New York Institute of Technology, PO Box 8000, Northern Boulevard, Old Westbury, NY 11568, USA; pdouris@nyit.edu

Accepted 8 April 2003

\begin{abstract}
Objectives: To quantify and compare fitness levels of middle aged practitioners of soo bahk do (SBD; a Korean martial art similar to karate) with those of sedentary subjects.

Methods: Eighteen volunteers, 14 men and four women (aged 40-60 years), participated. Nine sedentary subjects (mean age 46.7 years) and nine SBD practitioners (mean age 46.8 years) were matched for sex and age. All subjects participated in a one day battery of fitness tests. The following dependent variables were tested: body composition, balance, flexibility, quadriceps strength, grip strength, muscle endurance, and aerobic capacity.

Results: All dependent variables were analysed using paired $t$ tests. Body composition (\% body fat) for the SBD group was $18.9 \%$ v $30.8 \%$ for the sedentary group $(p=0.004)$. The SBD group was able to balance for 61.8 seconds $v 26.2$ seconds for the sedentary group $(p=0.02)$. The result for the sit and reach flexibility test was $22.3 \mathrm{~cm}$ for the SBD group $v 10.4 \mathrm{~cm}$ for the sedentary group $(p=0.01)$. The number of push ups performed in one minute was 47.0 for the SBD group $v 18.6$ for the sedentary group $(p=$ 0.0003), and the number of sit ups performed was 66.1 for the SBD group and 37.3 for the sedentary group ( $=0.00006$ ). Aerobic capacity was $41.0 \mathrm{ml} / \mathrm{kg} / \mathrm{min} v 31.1 \mathrm{ml} / \mathrm{kg} / \mathrm{min}$ for the sedentary group $(p=0.04)$. Quadriceps strength was $99.5 \%$ (peak torque/body weight) $\vee 83.0 \%$ for the sedentary group $(p=0.02)$. Only grip strength was not significantly different.

Conclusions: There were significant differences between the groups for most of the physical fitness tests. The SBD practitioners displayed greater aerobic capacity, balance, flexibility, muscle endurance, and strength, and less body fat than the sedentary controls matched for age and sex. SBD can be considered an excellent form of exercise for the promotion of fitness in adults. Health professionals should be aware that there are alternative methods to traditional exercise that can increase the physical fitness and health of the middle aged population.
\end{abstract}

M any studies have shown that practicing martial arts provides various physiological benefits. ${ }^{1-13}$ These include increases in strength, anaerobic capacity, balance, and flexibility as well as an overall improvement in cardiorespiratory fitness. Martial art is a general term that includes many forms and styles. Among the most popular are karate, judo, soo bahk do, tae kwon do, kung fu, and tai chi.

According to data from the American College of Sports Medicine (ACSM), 60\% of Americans are not regularly physically active, and $25 \%$ are completely sedentary. ${ }^{14}$ Risk factors related to a sedentary lifestyle include increased fat deposition and weight gain and decreased functional aerobic capacity, bone density, and mass. ${ }^{14}$

Participation in a regular exercise programme has been shown to be an effective intervention to reduce or prevent a number of functional declines associated with aging. Fitness training according to the ACSM should include exercises to improve the cardiorespiratory system, muscular strength, muscular endurance, and flexibility and maintain optimal body composition. ${ }^{14-16}$

There are many forms of martial arts, originating from different countries such as Japan, Korea, and China. Although they contain similar features, they each emphasise different systems of actions. ${ }^{17}$ Judo uses gripping and throwing techniques. Kung $\mathrm{fu}$ and tai chi use slow, purposeful, circular movements, which characterise it as a soft style. Karate and tae kwon do incorporate straight line powerful blocking, kicking, punching techniques as a major component, which characterise it as a hard style. Soo bahk do (also known as tang soo do) emphasises a combination of the hard styles of karate and tae kwon do with the soft style of the Chinese martial arts. Their system of action and the characteristic use of bare hands and feet as striking weapons are different from other martial art systems. ${ }^{17}$ Soo bahk do (SBD) involves a combination of both the soft and hard styles of other martial arts. The primary purpose and philosophy of SBD is rejuvenation and the prolonging of life beyond the normal span. ${ }^{17}$

There have been numerous studies investigating the short and long term benefits of martial art training. Karate training has been shown to be sufficient stimulus for improving aerobic power..$^{2-46712}$ Long term studies on elderly tai chi practitioners compared with sedentary control subjects showed the effectiveness of tai chi in improving cardiorespiratory function, flexibility, balance, muscle strength, and muscle endurance and decreasing body mass. ${ }^{35-79}$ Physiological profiles of elite young tae kwon do practitioners have shown them to possess above average anaerobic abilities, aerobic fitness, strength, and flexibility and low levels of body fat compared with population norms. ${ }^{2}$

Previous research has used subjects that have been martial artists either over the age of 65 or between the ages of 16 and 23. We found no study that documented the effects of martial art training on middle aged subjects (40-60 years of age). The primary purpose of this study was to document and compare the effects of SBD training compared with a sedentary lifestyle on body composition, muscle strength, muscle endurance, balance, flexibility, and aerobic capacity in middle aged people.

\section{METHODS}

Eighteen people volunteered to participate: nine sedentary subjects and nine martial art practitioners (seven men and two women in each group). Subjects were volunteers from a SBD school, community members, and members of the New York Institute of Technology. The institutional review board 
at the New York Institute of Technology approved the study, and all subjects read and signed an informed consent form before participating in the study. The inclusion criteria were: (a) subjects to be aged 40-60; $(b)$ subjects to present written medical clearance; $(c)$ the two groups to be matched by age and sex; (d) SBD practitioners to have been practicing for at least three years a minimum of twice a week for one hour a day; (e) SBD practitioners to participate in SBD only and no other formal physical exercise; $(f)$ sedentary/physically inactive subjects to not participate in any formal programme of physical exercise other than activities that are functionally and occupationally related. The exclusion criteria were: $(a)$ volunteers who did not fit the inclusion criteria; $(b)$ using the ACSM's guidelines, volunteers who presented with one or more of the following risk factors for coronary artery disease ${ }^{14}$ : family history; current cigarette smoking; hypertension (blood pressure $>140 / 90 \mathrm{~mm} \mathrm{Hg}$ or receiving antihypertensive medication); hypercholesterolaemia (total serum cholesterol $>2 \mathrm{~g} / \mathrm{l}$ or high density lipoprotein $<350 \mathrm{mg} / \mathrm{l}$ ); diabetes mellitus.

All subjects were tested between 9 am and 12 pm. Resting blood pressure, heart rate, height, and weight were recorded for all subjects before fitness testing. The following tests of physical fitness were administered in the following order on the same day and were completed within a two hour time period.

\section{Body composition}

A Lange skinfold caliper (Beta Technology, Cambridge, Maryland, USA) was used to measure subcutaneous fat at selected sites on the right side of the body. Sites measured in the men were the triceps, suprailiac, abdomen, and thigh, and those measured in the women were the triceps, suprailiac, and abdomen. ${ }^{14}$ Guidelines used to obtain the measurements were as follows: $(a)$ the skin fold was grasped firmly by the thumb and forefinger; $(b)$ the caliper was applied to the skinfold, and after two seconds the caliper dial was read; $(c)$ the reading was recorded in $\mathrm{mm}$; $(d)$ this procedure was repeated three times at all sites; (e) after all measurements were collected and summed, body fat percentage was calculated using equations given by Jackson and Pollack. ${ }^{18}$ The percentages were then used for data analysis.

\section{Balance}

Balance control was assessed by a single limb stance timed test with the eyes closed. ${ }^{3}{ }^{19}$ During the test, subjects were asked to stand on a firm surface and look straight ahead. The stopwatch began after the subject raised one leg and closed their eyes. The stopwatch was stopped when the subject became unstable and placed the flexed leg on the ground or at the completion of 60 seconds. Both legs were tested in random order. Total time for both legs was summed and documented and then used for data analysis.

\section{Strength}

Handgrip strength was measured with the Jamar handgrip dynamometer (Sammons Preston, Bolingbrook, Illinois, USA). The dominant hand was used for testing. Hand dominance was ascertained from the subject. The position used was $90^{\circ}$ of shoulder flexion with the elbow extended..$^{20}$ After one practice trial of a firm grip, the subject was asked to grip firmly and release. The measurement was recorded in $\mathrm{kg}$. Subjects were told to grip the dynamometer and give a maximal effort for three seconds. Three repetitions were performed. The best of the three scores was recorded and divided by the participant's total body weight to give a percentage. This percentage was then used for data analysis.

Quadriceps strength ${ }^{5} 6$ of the dominant leg was measured using a Cybex Norm II Isokinetic machine (Lumex Company,
Ronkonkoma, New York, USA). Leg dominance was identified by asking the subject to kick a ball. Preceding each test, a brief warm up was performed to familiarise subjects with the motion and to warm up the muscles before maximal output. Subjects were asked to perform five repetitions. Subjects were observed during warm up to ensure that they performed the exercise properly. They were sitting with $90^{\circ}$ of hip flexion. Subjects were attached in a position after adjustment of the height of the dynamometer and the length of the support lever, allowing the axis of rotation of the dynamometer to be aligned with the subject's knee joint. Each subject was stabilised at the chest, waist, and thigh with a strap. A shin strap was secured to the lower leg above the malleoli. The test was performed on the subject's dominant lower limb. The Cybex Norm was calibrated according to the manual before each test day. Each subject was asked to perform one isometric contraction for the knee extensors at $45^{\circ}$ less than from full extension. Subjects were instructed to extend the knee as far as possible to produce a maximum isometric contraction of the quadriceps. The measurements were obtained in ft-lbs. The exercise was repeated five times. All subjects were allowed a two minute rest period between tests. Of the five scores obtained, the highest peak torque was divided by the participant's body weight to give a percentage, which was used for data analysis.

\section{Endurance}

Muscle endurance was assessed using the one minute timed test for bent knee sit ups and push ups. ${ }^{14}$ For the sit ups, subjects were lying in a supine position with both knees bent at right angles and both feet were shoulder width apart. Both arms were placed at the side of the trunk. Two pieces of tape were placed on the mat to ensure that a proper sit up was performed. The first tape was placed at the tip of the middle finger of both hands, and the second tape was placed 3 inches away from the first tape. The subjects were asked to touch the second tape with their middle finger and return to the starting position (shoulders touching the floor); this constituted a sit up. All subjects were asked to perform as many sit ups as possible within one minute. A rocking or bouncing movement was not permitted. The buttocks remained in contact with the mat at all times. The total numbers of sit ups were documented and used for data analysis.

The procedure for push ups differed for men and women. The men lay on their stomach with both legs together. The hands were pointing forward and positioned under the shoulders. The subjects performed push ups from the mat by fully straightening the elbows and using their toes as a pivotal point. The upper body was kept in a straight line. Subjects returned to the starting point with chin to the mat. The stomach and thigh were not allowed to touch the mat. The women used the same procedure except that the knees were bent and the ankles crossed over and plantar flexed. The knees were the pivotal point. All subjects performed as many push ups as possible within one minute. The total numbers of push ups were documented and used for data analysis.

\section{Flexibility}

A sit and reach flexibility box (Novell Products, Rockton, Illinois, USA) was used to assess low back and hip joint flexibility. ${ }^{14}$ Each subject sat with legs fully extended with the bare soles of the feet placed flat against the flexibility box. With the knees fully extended, arms evenly stretched, palms down, the participant reached forward without jerking. The subject pushed the sliding marker along the scale with the fingertips as far as possible. The position of maximum trunk flexion was held for about two seconds. The test was repeated three times. The best value in $\mathrm{cm}$ was documented and used for data analysis. 


\section{$\mathrm{Vo}_{2} \mathrm{MAX}$}

A Sensormedics Horizon Treadmill (Anaheim, California, USA) was used to assess $\mathrm{VO}_{2}$ MAX using a submaximal graded exercise test. Each stage lasted three minutes. One MET increments were used until $70 \%$ of age predicted maximal heart rate was attained using Karvonen's heart rate reserve formula. Heart rate was measured using a Polar Beat Monitor (Polar Electro Inc, Woodbury, New York, USA) to ensure validity and reliability. Heart rate was recorded three times per stage, and blood pressure, at the rate of perceived exertion, was recorded once during each stage. The test was terminated when the patient achieved the predetermined heart rate or met any other test termination criteria outlined by the ACSM guidelines. ${ }^{14}$ The subject then performed an active cool down to return the blood pressure to within $10 \mathrm{~mm} \mathrm{Hg}$ of the resting value and heart rate to under 100 beats/min. Heart rate was measured every minute of cool down, with blood pressure measured every other minute. The $\mathrm{VO}_{2} \mathrm{MAX}$ expressed in $\mathrm{ml} / \mathrm{kg} / \mathrm{min}$ was determined by plotting a line between the heart rate measured during the last minute of each stage. This line was extrapolated to age predicted maximal heart rate, and a perpendicular line was dropped to the $\mathrm{x}$ axis to estimate the work rates that would have been achieved if the person had worked to maximum. ${ }^{14}$ This value was used for data analysis.

\section{Statistical analysis}

This was an experimental study. The independent variables were the martial art SBD and the sedentary lifestyle. The dependent variables were the $\mathrm{VO}_{2} \mathrm{MAX}$, sit and reach test, quadriceps strength, grip strength, body composition, push ups, sit ups, and balance. In this study, the eight dependent variables are independent measures of the construct of physical fitness; they do not correlate and cannot be substituted for one another. Therefore, the results were analysed using paired $t$ tests for each dependent variable, with significance set at the 0.05 level.

In the light of controversy about the role of correction for multiple comparisons, Senghas ${ }^{21}$ suggests presenting the calculation of the upper limit of $\alpha$. The upper limit of $\alpha$ for this study was calculated at 0.34 . This translates into a $34 \%$ chance that at least one comparison may appear to show a significant difference when in reality there is no significant difference.

\section{RESULTS}

Table l summarises the physiological characteristics of the subjects. The physiological data for the nine sex and age matched pairs were similar. The SBD practitioners had been practicing for at least three years (mean (SD) 6.2 (3.1) years) a minimum of twice a week.

The SBD practitioners were significantly different from the sedentary group in all fitness tests studied in all the dependent variables except grip strength (table 2).

\begin{tabular}{|c|c|c|}
\hline & Soo Bahk Do & Sedentary \\
\hline Age & $46.8(4.2)$ & $46.7(3.7)$ \\
\hline Height $(\mathrm{cm})$ & $170.7(8.9)$ & $174.1(7.6)$ \\
\hline Weight (kg) & $80.9(14.9)$ & $87.8(15.9)$ \\
\hline Resting HR (beats/min) & $69.9(8.7)$ & $71.6(8.6)$ \\
\hline \multicolumn{3}{|l|}{ Resting BP $(\mathrm{mm} \mathrm{Hg})$} \\
\hline Systolic & $117.4(10.1)$ & $113.9(18.8)$ \\
\hline Diastolic & $73.3(12.3)$ & $70.8(10.8)$ \\
\hline
\end{tabular}

\section{DISCUSSION}

The primary finding of this study was that middle aged adults whose only exercise programme is SBD, who have participated at least twice a week for at least three years display greater cardiorespiratory fitness, muscle strength, muscle endurance, flexibility, and balance, and less body fat than matched healthy sedentary subjects. According to the ACSM, "health-related physical fitness is a state characterised by an ability to perform daily activities with vigour, and a demonstration of traits and capacities that are associated with low risk of premature development of the hypokinetic diseases". ${ }^{14}$ Developing and maintaining fitness includes exercises that address cardiorespiratory endurance, optimal body composition, muscle strength and endurance, flexibility and balance in the older adult. ${ }^{15} 16$ Incorporating all components of fitness is associated with a lower risk of developing disease and functional disability. ${ }^{14} 16$

A typical SBD class is one hour in length. All classes were led by a certified instructor of the United States Soo Bahk Do Moo Duk Kwan Federation. The class usually includes a 15 minute warm up of calisthenics, flexibility exercises, punching, blocking, and kicking drills. The core of the class may include an additional 10 minutes of basic blocking, kicking, and punching drills, 20 minutes of hyung (set forms in pre-established sequences of defensive and offensive techniques and movements), 10 minutes of sparring (the execution of defensive and offensive techniques while freely moving against an opponent), and self defence techniques. The class typically ends with a five minute cool down period of additional flexibility exercises.

Imamura et $a l^{4}$ have shown that the energy expenditure of a typical karate class, similar to a SBD class, is above the accepted threshold for fat weight loss and for increasing $\mathrm{VO}_{2} \mathrm{MAX}$. The performance of karate kata or hyungs has been shown to be an effective means of improving aerobic power. $^{8}{ }^{12}$ SBD and karate both contain the hard style training of punching, kicking, and sparring. However, some of the SBD hyungs contain the slower and circular motions similar to the soft styles of the Chinese arts of kung fu and tai chi. These slower and circular motions have been associated with the improved postural stability and aerobic power of older tai chi practitioners. ${ }^{3679}$ A direct comparison of energy expenditure between karate and tai chi training shows 10 METS (maximal intensity) and 4 METS (moderate intensity) respectively. METS refer to the multiples of metabolic equivalent used for classification of exercise intensity.22 Although there are no data on the energy cost of SBD training, as the training is similar to karate, it is assumed that the energy cost would be similar.

The development and maintenance of cardiorespiratory fitness has been well documented to be associated with various health benefits and a reduction in disease risk factors. ${ }^{14-16}$ The SBD practitioners displayed significantly greater $\mathrm{VO}_{2} \mathrm{MAX}$ than matched sedentary controls. $\mathrm{VO}_{2} \mathrm{MAX}$ is the accepted measure of cardiorespiratory fitness. ${ }^{14}$ Lan et al in a cross sectional study reported that geriatric male tai chi practitioners had a $19 \%$ greater $\mathrm{VO}_{2} \mathrm{MAX}$, and female tai chi practitioners had $18 \%$ greater $\mathrm{VO}_{2} \mathrm{MAX}$, than their sedentary counterparts. A 12 month tai chi training programme increased aerobic power by $18.1 \%$ in elderly men and by $21.3 \%$ in elderly women. ${ }^{6}$ Heller $e^{2} a^{2}$ reported that young tae kwon do practitioners had $20 \%$ greater $\mathrm{VO}_{2} \mathrm{MAX}$ than population norms. In the present study, the SBD practitioners displayed $31.8 \%$ greater $\mathrm{VO}_{2} \mathrm{MAX}$ than the sedentary group. The SBD group scored in the above average centile ranking for maximal aerobic power while the sedentary group was ranked below average, according to the ACSM. ${ }^{14}$ The training intensity of SBD appears to provide adequate 
Table 2 Comparison of results of fitness tests between the soo bahk do practitioners and the sedentary subjects

\begin{tabular}{llllll}
\hline & Soo bahk do & Sedentary & $\boldsymbol{t}$ Value & $\begin{array}{l}\text { Percentage } \\
\text { difference }\end{array}$ & $\mathbf{p ~ V a l u e ~}$ \\
\hline Balance (seconds) & $61.8(37.8)$ & $26.2(16.6)$ & 2.64 & 135.8 & 0.02 \\
Vo ${ }_{2} \mathrm{MAX}(\mathrm{ml} / \mathrm{kg} / \mathrm{min})$ & $41.0(8.9)$ & $31.1(12.3)$ & 2.02 & 31.8 & 0.04 \\
Quad strength (\% kg/BW) & $99.5(11.3)$ & $83.0(15.5)$ & 2.44 & 19.9 & 0.02 \\
Grip strength (\% kg/BW) & $59.4(12.6)$ & $51.5(9.9)$ & 1.47 & 15.3 & 0.09 \\
Sit and reach (cm) & $22.2(12.0)$ & $10.4(9.4)$ & 2.72 & 113.5 & 0.01 \\
Sit ups & $66.1(12.9)$ & $37.3(12.4)$ & 9.37 & 77.2 & 0.00006 \\
Push ups & $47.0(10.9)$ & $18.6(9.6)$ & 5.4 & 152.6 & 0.0003 \\
Body composition (\%) & $18.9(4.5)$ & $30.8(8.4)$ & 3.58 & 63.0 & 0.004 \\
\hline Values are mean (SD). & & & & & \\
BW, Body weight. & & & & &
\end{tabular}

stimulus to maintain cardiorespiratory fitness in middle aged practitioners.

The decline in muscle strength and endurance contributes to decreases in functional capacities associated with aging. ${ }^{15}$ Our result showing $19.9 \%$ greater quadriceps strength in the SBD group compares favourably with other studies. ${ }^{25}{ }^{6}$ Heller et $a l^{2}$ reported that young tae kwon do practitioners displayed $27 \%$ greater quadriceps strength than population norms. Lan et $a l^{5}$ reported quadriceps strength changes in elderly men and women of $18.3 \%$ and $20 \%$ respectively after a six month tai chi training programme. Similar results were reported by Lan et $a l^{6}$ after a 12 month tai chi training programme. Martial art training appears to improve quadriceps strength by its action of performing kicks, punches, and blocks in various degrees of knee flexion in a closed kinematic chain.

Grip strength has been shown to be a predictor of mortality and functional decline. ${ }^{23}$ Although the SBD practitioners displayed greater grip strength $(59.4 \%$ v $51.5 \%$ (kg/body weight)), the difference was not significant. Both groups scored within normative ranges for their sex and age. ${ }^{20}$ Future research should address the effects of martial art training on grip strength in the older practitioner.

Muscle endurance is the ability of a muscle group to execute contractions over a period of time sufficient to cause muscular fatigue. ${ }^{14}$ The push up is a simple field test to evaluate the endurance of the upper body. ${ }^{14}$ The SBD practitioners displayed significantly greater upper body endurance by performing an average of 47.0 push ups compared with 18.6 for the sedentary group, a $153 \%$ difference. The sit up evaluates the endurance of the abdominal muscle groups. The SBD practitioners averaged 66.1 sit ups compared with 37.3 sit ups for the sedentary group, a 77\% difference. SBD appears to offset the loss of muscle strength and endurance that is associated with normal aging in this middle aged group.

Flexibility is the ability of a joint or multiple joints to move through the complete range of motion. ${ }^{14}$ Loss of flexibility can significantly impair a person's ability to perform exercise and accomplish activities of daily living as well as other important functional activities. It is well documented that flexibility declines with age. ${ }^{15}$ The sit and reach test is commonly used to assess hamstring, low back, and hip joint flexibility. ${ }^{14}$

The SBD practitioners were significantly more flexible than their sedentary counterparts, as is evident in the $114 \%$ difference between the groups $(22.2 v 10.4 \mathrm{~cm})$. Young tae kwon do practitioners displayed similar results in comparison with normative data. ${ }^{2}$ Our results are also comparable to those of Lan et $a l^{7}$ and Hong et al, ${ }^{3}$ who in cross sectional studies have shown elderly tai chi practitioners to have greater trunk flexibility than matched sedentary controls. The greater flexibility of the SBD practitioners may be attributed to the stretching exercises and postures found in a typical SBD class.
Balance or postural stability has been defined as the ability to maintain equilibrium in a gravitational field by keeping or returning the centre of body mass over its base of support. There is overwhelming evidence that postural stability declines with age and that poor postural stability or balance is associated with frequent falling. ${ }^{15}$ The SBD practitioners displayed far superior balance over their sedentary counterparts: $61.8 v 26.2$ seconds, a $136 \%$ difference. Tai chi for the elderly has been shown to improve postural stability ${ }^{159}$ and has been recommended by the American College of Medicine ${ }^{15}$ and Wolf et $a l^{10}$ as an appropriate intervention for improving postural stability in the elderly. Our results are in disagreement with those of Tse and Bailey, ${ }^{9}$ who reported significant differences in one legged balance with eyes open but not eyes closed between geriatric tai chi practitioners and sedentary controls. Our results are similar to those of Hong et al, ${ }^{3}$ who showed that older tai chi practitioners obtained higher scores for one legged balance with eyes closed than sedentary controls. The SBD practitioners displayed greater balance than their middle aged sedentary counterparts; these results suggest SBD to be an alternative intervention to tai chi for improving postural stability and balance.

Body composition refers to the percentage of body weight that is fat and fat-free tissue. ${ }^{14}$ Excess body fat is associated with hypertension, hyperlipidaemia, type 2 diabetes, complex and cardiovascular disease. ${ }^{14-16}{ }^{21}$ A sedentary lifestyle and the aging process appear to negatively affect body composition by the accumulation of fat and the concomitant loss of lean muscle mass. The SBD group had a significantly lower percentage of body fat than the sedentary group (18.9\% $v$ $30.8 \%$ ). These results imply that SBD training was beneficial to body composition, which may be attributed to the effects of muscle mass preservation and body fat reduction. According to ACSM fitness standards, SBD practising men attained the 70th centile, SBD practising women the 60th centile, whereas their sedentary counterparts were in the 10th centile. Geriatric tai chi practitioners ${ }^{7}$ and young tae kwon do practitioners ${ }^{2}$ displayed similar favourable body composition results when compared with sedentary controls and normative data.

Our study has shown that the long term practice of SBD may benefit cardiorespiratory function, muscle strength, muscle endurance, flexibility, balance, and body composition in middle aged practitioners. Similar physiological benefits have been documented in young ${ }^{2}$ and older martial artists. ${ }^{135-7911}$ The results provide evidence that SBD training is an effective intervention that may reduce or prevent a number of functional and physiological declines associated with normal aging. In addition, the exercise characteristics of SBD fulfil the criteria proposed by the ACSM for developing and maintaining optimal body composition, flexibility, balance, and cardiorespiratory and muscular fitness in healthy adults ${ }^{15}{ }^{16}$ Future studies may take a 


\section{Take home message}

Health professionals should be aware that alternative methods, such as martial arts training, can increase physical fitness of middle aged people. Middle aged soo bahk do practitioners displayed greater aerobic capacity, balance, flexibility, muscle endurance, and strength, and less body fat than sedentary counterparts.

longitudinal approach rather than the cross sectional approach. Length of training should also be controlled in order to document the physiological changes during specific time periods. This study should be replicated with younger and older practitioners with larger samples. It appears that SBD training may be a complete form of exercise encompassing all the components of fitness. A comparison with other forms of exercise and martial arts is warranted. These results suggest that health professionals should be aware of alternative modes of exercise delivery for increasing the physical fitness and health of the middle aged population.

\section{Authors' affiliations \\ P Douris, A Chinan, M Gomez, A Aw, D Steffens, S Weiss, New York Institute of Technology, Old Westbury, NY, USA}

\section{REFERENCES}

1 Hain TC, Fuller L, Weil L, et al. Effects of tai chi on balance. Arch Otolaryngol Head Neck Sur 1999; 125:1191-5.

2 Heller J, Peric T, Dlouha R, et al. Physiological profiles of male and female tae kwon-do black belts. J Sports Sci 1998;16:243-9

3 Hong Y, Li XJ, Robinson PD. Balance control, flexibilty, and cardiorespiratory fitness among older tai chi practitioners. Br J Sports Med 2000;34:24-9.

4 Imamura H, Yoshimura Y, Nishimura S, et al. Oxygen uptake, heart rate and blood lactate responses during and following karate training. Med Sci Sports Exerc 1999:31:342-7.

5 Lan C, Lai JS, Chen SY, et al. Tai chi chuan to improve muscular strength and endurance in elderly individuals: a pilot study. Arch Phys Med Rehabil 2000;81:604-7.

6 Lan C, Lai JS, Chen SY, et al. 12-Month tai chi training in the elderly: its effect on health and fitness. Med Sci Sports Exerc 1998;30:345-51.

7 Lan C, Lai JS, Wong MK, et al. Cardiorespiratory function, flexibility, and body composition among geriatric tai chi chuan practitioners. Arch Phys Med Rehabili 1996;77:612-6.

8 Shaw DK, Deutsch DT. Heart rate and oxygen uptake response to performance of karate kata. J Sports Med 1982;22:461-8.

9 Tse SK, Bailey DM. Tai chi and postural control in the well elderly. Am J Occup Ther 1992;46:295-300

10 Wolf S, Coogler C, Xu T. Exploring the basis for tai chi chuan as a therapeutic exercise approach. Arch Phys Med Rehabili 1997;78:886-92.
11 Young DR, Appel $\sqcup$, Jee $S$, et al. The effects of aerobic exercise and tai chi on blood pressure on older adults. J Am Geriatr Soc 1999;47:277-84.

12 Zehr EP, Sale DG. Oxygen uptake, heart rate and blood lactate reponses to the chito-ryu seisan kata in skilled karate practitioners. Int J Sports Med 1993; 14:269-74.

13 Zehr EP, Sale DG, Dowling JJ. Ballistic movement performance in karate athletes. Med Sci Sports Exerc 1997;29:1366-77.

14 American College of Sports Medicine. ACSM'S resource manual for guidelines for exercise testing and prescription, 6th ed. Philadelphia: Lippincott Williams and Wilkins, 2001:57-90.

15 American College of Sports Medicine Position Stand. Exercise and physical activity for older adults. Med Sci Sports Exerc 1998;30:992-1008.

16 American College of Sports Medicine Position Stand. The recommended quality, and quality of exercise for developing, and maintaining cardiorespiratory, and muscular fitness, and flexibility in adults. Med Sci Sports Exerc 1998;30:975-91.

17 Kee H. Soo Bahk Do Tang Soo Do, Volume 1. Springfield, NJ: Paragon Press, 1995:24-30.

18 Jackson AS, Pollack ML. Practical assessment of body composition. Phys Sportsmed 1985; 13:76-90.

19 Rikli RE, Edwards DJ. Effects of three year exercise program on motor function and cognitive processing speed in older woman. Res $Q$ Exerc Sport $1991 ; 62: 61-7$

20 Mathiowetz VN, Kashman G, Volland K, et al. Grip and pinch strength: normative data for adults. Arch Phys Med Rehabil 1985;85:69-74.

21 Senghas RE. Statistics in the Journal of Bone and Joint Surgery: suggestions for authors. J Bone Joint Surg [Am] 1992;74:319-20.

22 American College of Sports Medicine. ACSM'S resource manual for guidelines for exercise testing and prescription, 4th ed. Philadelphia: Lippincott Williams and Wilkins, 2000:673-86.

23 Bohannon RW. Dynamometer measurements of hand grip strength predict multiple outcomes. Percept Mot Skills 2001 ;93:323-8.

\section{COMMENTARY}

This paper adds to the evidence that exercise provides important benefits to those who participate on a regular basis. Risk factors associated with a sedentary lifestyle are understood by many members of society but often are not acted on. One of the characteristics of exercise programmes should be that the participants enjoy the experience. The programme of exercise and associated activities noted in the paper about soo bahk do meet this criterion. The paper clearly shows the physiological benefits to the exercise group in this study. The authors make an important point in their statistical analysis alerting the reader to the chance of a significant difference being shown that could be incorrect. The authors in the discussion point out the benefits of fitness in an elderly population, with the benefits extending to areas of postural stability and balance as well.

J Echternach Old Dominion University, William B Spong Jr Hall, Norfolk, VA 23539-0288, USA; jechtern@odu.edu 